\title{
An Exact Performance Analysis of MRC / OSTBC over Generalized Fading Channels
}

\author{
George A. Ropokis, Athanasios A. Rontogiannis, Member, IEEE, P. Takis Mathiopoulos, Senior Member, IEEE, \\ and Kostas Berberidis, Senior Member, IEEE
}

\begin{abstract}
A unified analytical framework for evaluating the performance of maximal ratio combining (MRC) and orthogonal space-time block coding (OSTBC) over generalized fading channels is presented. The basic motivation for developing such a framework pertains to analyzing the distribution of a sum of squared random variables (RVs) belonging to different families of fading distributions. Following a novel analytical approach stemming from the definition of a common moment generating function (MGF) model for these families of distributions, the probability density function (PDF) and the cumulative distribution function (CDF) of a general sum of squared RVs are expressed by simple infinite Gamma series expansions. Based on these expressions, the capacity and error probability of MRC/OSTBC over generalized fading channels is thoroughly studied. The developed theory is used to evaluate the performance of OSTBC for a mixed Nakagami- $m /$ Rice fading model and novel analytical results are presented. The accuracy of the derived expressions is validated by extensive Monte Carlo simulations.
\end{abstract}

Index Terms-Diversity methods, fading channels, OSTBC, MRC, performance analysis.

\section{INTRODUCTION}

D UE to their ability to effectively combat fading, diversity combining techniques have been used extensively in the past to improve the performance of wireless and mobile communications systems [1]. Among the various diversity combining techniques, recently maximal ratio combining (MRC) and orthogonal space-time block coding (OSTBC) have gained particular interest [2]. Although both techniques require increased implementation complexity due to the need for accurate channel estimation, they can deliver maximal diversity by suitably combining different replicas of the transmitted and/or the received signal. When MRC or OSTBC is employed the signal to noise ratio (SNR) at the receiver is expressed as a sum of squared random variables (RVs). Hence, it makes sense to analyze the performance of such systems

Paper approved by G. M. Vitetta, the Editor for Equalization and Fading Channels of the IEEE Communications Society. Manuscript received May 25, 2009; revised October 26, 2009.

This paper is part of the 03ED939 research project, implemented within the framework of the "Reinforcement Programme of Human Research Manpower" (PENED) and co-financed by National and Community Funds (75\% from E.U.-European Social Fund and 25\% from the Greek Ministry of Depelopement-General Secretariat of Research and Technology).

G. A. Ropokis is with the Dept. of Computer Engineering \& Informatics, University of Patras, 26500, Rio, Greece, and the Institute for Space Applications and Remote Sensing, National Observatory of Athens, 15236, Athens, Greece (e-mail: ropokis@space.noa.gr).

A. A. Rontogiannis and P. T. Mathiopoulos are with the Institute for Space Applications and Remote Sensing, National Observatory of Athens, 15236, Palea Penteli, Athens, Greece (e-mail: \{tronto, mathio\}@space.noa.gr).

$\mathrm{K}$. Berberidis is with the Dept. of Computer Engineering \& Informatics, University of Patras, 26500, Rio, Greece (e-mail: berberid@ceid.upatras.gr).

Digital Object Identifier 10.1109/TCOMM.2010.09.090282 by directly employing the probability density function (PDF) and the cumulative distribution function (CDF) of the sum of squared RVs. Moreover, it is desirable to express these distribution functions in the simplest possible form, so as to facilitate the calculation of several performance metrics, such as for example symbol and bit error probability (SEP and BEP) or capacity.

A simple, yet accurate, representation for the PDF of the sum of squared RVs belonging to the same family of distributions $^{1}$ is based on suitable infinite series expansions. In early works that have appeared in the statistical literature [3],[4] the distribution of the sum of squared Gaussian RVs has been studied and several series expansions for the PDF have been derived. More recently, using a Gamma series representation, the PDF and CDF of the sum of independent Gamma, i.e., squared Nakagami RVs, have been derived in [5]. Based on these expressions the performance of MRC over Nakagami fading channels has been studied in [6]. In [7], the Gamma series representation has been adopted for the performance analysis of OSTBC over Hoyt fading channels, while in [8] the same series expression has been applied for computing matched filter bounds for binary phase shift keying (BPSK) over multipath Rician fading channels. Using a Gamma series representation, the capacity of MRC over Rician and Hoyt fading channels has been studied in [9]. Capacity and error probability analysis of OSTBC for independent identically distributed (i.i.d.) Rayleigh, Nakagami- $m$ and Rice fading channels has been presented in [10] and outage performance analysis of OSTBC over Ricean fading channels using either a power or a Laguerre series representation for the PDF of the SNR has been developed in [11].

In several applications, the fading coefficients corresponding to the different channels of an MRC combiner or an OSTBC scheme may not be identically distributed nor even belong to the same family of distributions. Such a generalized fading model has been considered in [12], where by applying a numerical inversion of the Laplace transform of the CDF, a moment generating function (MGF) based numerical technique has been proposed for evaluating the outage probability of MRC. More recently, a similar problem has also been addressed in [13], where a simple and efficient algorithm for numerically inverting the MGF of the SNR for OSTBC over generalized fading channels has been presented. However, to the best of our knowledge, an exact performance analysis for both the capacity and the error probability of MRC/OSTBC over such generalized fading channels, which avoids numerical

\footnotetext{
${ }^{1}$ In other words, the sum contains RVs having the same PDF possibly with different parameters.
} 
TABLE I

MGFS OF THE FADING CHANNELS UNDER CONSIDERATION

\begin{tabular}{|c|c|c|}
\hline Channel model & Fading Parameters & $\overline{\text { MGF }}$ \\
\hline Nakagami- $m$ & $\Omega_{j}, m_{j} \geq 0$ & $M_{X_{j}^{2}}^{N}(s)=\frac{1}{\left(1-s \frac{\Omega_{j}}{m_{j}}\right)^{m_{j}}}$ \\
\hline Rice & $\Omega_{j}, K_{j} \geq 0$ & $M_{X_{j}^{2}}^{R}(s)=\frac{1}{1-s \frac{\Omega_{i}}{K_{j}+1}} \exp \left(\frac{\frac{\sigma_{j}+1}{K_{j}+1}}{1-s \frac{\Omega_{j}}{K_{j}+1}}\right)$ \\
\hline Hoyt & $\Omega_{j} \geq 0,0 \leq q \leq 1$ & $\begin{aligned} M_{X_{j}^{2}}^{H}(s) & =\frac{1}{\sqrt{\left(1-\frac{2 \Omega_{j} q_{j}^{2}}{1+q_{j}^{2}} s\right)\left(1-\frac{2 \Omega_{j}}{1+q_{j}^{2}} s\right)}} \\
M^{B}(s) & =\frac{\exp \left(\frac{\mu_{j, 1}^{2} \Omega_{j} s}{1-2 \sigma_{j, 1}^{2} \Omega_{j} s}+\frac{\mu_{j, 2}^{2} \Omega_{j} s}{1-2 \sigma_{j, 2}^{2} \Omega_{j} s}\right)}{}\end{aligned}$ \\
\hline Shadowed Rice & $\Omega_{j}>0, \mu_{j, 1}, \mu_{j, 2}, \sigma_{j, 1}, \sigma_{j, 2}$ & $\begin{array}{l}M_{X_{j}^{2}}^{2}(S)=\frac{\sqrt{\left(1-2 \sigma_{j, 1}^{2} \Omega_{j} s\right)\left(1-2 \sigma_{j, 2}^{2} \Omega_{j} s\right)}}{M_{X_{j}^{2}}^{S R}(s)=\frac{\left(1-2 \lambda_{j} s\right)^{m_{j}-1}}{\left(1-\left(2 \lambda_{j}+\frac{\Omega_{j}}{{ }^{2}}\right)_{s}\right)^{m_{j}}}}\end{array}$ \\
\hline
\end{tabular}

simplifications and approximations, does not exist in the open technical literature. This is due to the fact that such analysis becomes extremely complicated when there is a need to manipulate and integrate a number of different fading RVs.

In an effort to introduce a unified analytical framework, first a generalized fading model for MRC/OSTBC is defined and analyzed in this paper. In particular, we consider the very general case where the channel fading coefficients of an MRC/OSTBC scheme are independent and can follow different families of distributions, namely Rayleigh, Nakagami- $m$, Rice, Hoyt, Beckmann and shadowed-Rice (SR). By adopting the classical PDF method and using a novel analytical approach, new and simple to evaluate expressions for the $\mathrm{PDF}$ and CDF of the sum of squared RVs belonging to the set of distribution families mentioned before, are derived. To this end, the MGF of the sum is first expressed in a general compact form. Based on this expression, it is shown that, as in [3], [4] the PDF and CDF of the sum of squared RVs can be written in a simple Gamma series form. By employing these PDF and CDF expressions, a general framework for the performance analysis of MRC/OSTBC over generalized fading channels in terms of capacity and error probability for various modulation schemes is presented. Exact analytical expressions for the information outage probability, ergodic capacity, SEP and BEP are derived. To illustrate the validity of the proposed general framework, an OSTBC system operating in the presence of mixed Nakagami- $m$ /Rice fading is studied and novel performance analysis expressions are obtained. Efficient numerical solutions are also proposed to simplify computation of the more complex functions that appear in these final expressions. Complementary performance evaluation simulation results are presented, which completely corroborate the theoretical analysis.

The outline of the paper is as follows. After this introduction, in Section II the problem is formulated and the general expression of the MGF is presented. The PDF and CDF of the sum of squared RVs as Gamma series representations are derived in Section III. A general performance analysis in terms of capacity and error probability is presented in Section IV. An application of the proposed general analysis is then described in Section V. Various simulation results are presented in Section VI and concluding remarks are discussed in Section VII.

\section{Problem Formulation}

Let us consider $n$ independent RVs, $X_{j}, j=1,2, \ldots, n$, which are not necessarily identically distributed nor even follow the same distribution. By defining $Y$, as the sum of squares of $X_{j}$ 's, i.e.,

$$
Y \triangleq \sum_{j=1}^{n} X_{j}^{2}
$$

the MGF of $Y$ is given by

$$
M_{Y}(s)=\prod_{j=1}^{n} M_{X_{j}^{2}}(s)
$$

with $M_{X_{j}^{2}}(s)$ denoting the MGF of $X_{j}^{2}$. One of our main goals is the derivation of general expressions for the distribution (PDF and CDF) of $Y$, when $X_{j}$ 's follow a number of generic distributions that are commonly used to model fading in wireless communication channels. More specifically, in this analysis it is assumed that each $\mathrm{RV} X_{j}$ may follow one of the distributions shown in Table I, where besides the commonly used Nakagami- $m$, Rice and Hoyt fading distributions, the more complex Beckmann and Shadowed Rice (SR) distributions are also included. The Beckmann distribution is a rather general distribution that includes as special cases the Rayleigh, Rice and Hoyt distributions. The SR distribution is a very accurate fading model for land mobile satellite (LMS) communication channels [14], [15]. For all these distributions, the expressions of the MGFs have been conveniently rewritten so as to facilitate the subsequent analysis. Indeed, by assuming that each $X_{j}$ is distributed according to one of the above fading models, the MGF of $Y$ defined in (1) takes the following general form

$$
M_{Y}(s)=\prod_{i=1}^{n_{0}} \frac{\exp \left(\delta_{i} \frac{a_{i} s}{1-a_{i} s}\right)\left(1-b_{i} s\right)^{k_{i}}}{\left(1-a_{i} s\right)^{p_{i}}}
$$

with $n_{0} \geq n$, while the real and non-negative parameters $a_{i}, b_{i}, \delta_{i}, p_{i}, k_{i}\left(p_{i}>k_{i}\right)$ take appropriate values depending on the specific distributions of the individual $X_{j}$ 's. ${ }^{2}$ Based on the

\footnotetext{
${ }^{2}$ Note that $n_{0}>n$ whenever at least one of the $X_{j}$ 's is Beckmann or Hoyt distributed. Moreover, although $k_{i}$ may be negative (e.g. for SR RVs with $m_{j}<1$ ), in the analysis to follow it will be assumed that $k_{i} \geq 0$. This assumption will be made without any loss of generality since a similar analysis can be made for $k_{i}<0$.
} 
generic expression of the MGF given in (3), it will be shown that the classical approach developed for the distribution of quadratic forms in normal RVs [3], [4], can be extended to the more general problem considered in this paper. Applying a suitable transformation in the MGF, the PDF of $Y$ will be expressed in a simple Gamma series form, as will be described below.

\section{Distribution of The Sum OF Squared RVS}

Let $f_{Y}(y)$ be the PDF of $Y$, whose MGF is given by (3) with $a_{i}, b_{i}, \delta_{i} \geq 0$ and $m_{i}>k_{i} \geq 0$. As wellknown, $f_{Y}(y)$ is given by the inverse Laplace transform $\mathcal{L}^{-1}\left\{M_{Y}(-s)\right\}$. However, due to the form of $M_{Y}(-s)$ from (3), direct application of the inverse Laplace transform does not lead to a tractable closed-form expression for the PDF. To overcome this problem, $M_{Y}(-s)$ will be expanded as an infinite series comprising functions whose inverse Laplace transform is known. Similar to [3],[4], to accomplish this, the following transformation is used

$$
\theta(s)=\frac{1}{1+s \beta}
$$

where $\beta$ is an arbitrary positive parameter that controls the convergence of the PDF series which will be derived. Notice that for any $\alpha>0$ the following identities hold

$$
1+\alpha s=\frac{\alpha}{\beta \theta(s)}\left(1-\left(1-\frac{\beta}{\alpha}\right) \theta(s)\right)
$$

and

$$
\frac{\alpha s}{1+\alpha s}=1-\frac{\beta}{\alpha} \frac{\theta(s)}{1-\left(1-\frac{\beta}{\alpha}\right) \theta(s)} .
$$

Hence, by substituting (5) and (6) in (3), $M_{Y}(-s)$ is readily expressed as

$$
\begin{aligned}
M_{Y}(-s)= & A \exp \left(\theta(s) \sum_{i=1}^{n_{0}} \frac{\delta_{i} \beta}{a_{i}\left(1-\gamma_{i} \theta(s)\right)}\right) \theta^{\rho}(s) \\
& \times \prod_{i=1}^{n_{0}} \frac{\left(1-\zeta_{i} \theta(s)\right)^{k_{i}}}{\left(1-\gamma_{i} \theta(s)\right)^{p_{i}}}
\end{aligned}
$$

where

$$
A=\left(\prod_{i=1}^{n_{0}} \frac{b_{i}^{k_{i}}}{a_{i}^{p_{i}}} \beta^{p_{i}-k_{i}}\right) \exp \left(-\sum_{i=1}^{n_{0}} \delta_{i}\right), \quad \rho=\sum_{i=1}^{n_{0}}\left(p_{i}-k_{i}\right)
$$

and

$$
\gamma_{i}=1-\frac{\beta}{a_{i}}, \quad \zeta_{i}=\left\{\begin{array}{l}
1-\frac{\beta}{b_{i}}, b_{i} \neq 0 \\
0, b_{i}=0
\end{array}\right.
$$

The main idea here is to express $M_{Y}(-s)$ given in (7) as a series expansion with respect to $\theta(s)$ so that, through Laplace inversion, a simple formula for the PDF can be obtained. For this purpose, let us first introduce the function ${ }^{3}$

$$
L(\theta)=\exp \left(\theta \sum_{i=1}^{n_{0}} \frac{\delta_{i} \beta}{a_{i}\left(1-\gamma_{i} \theta\right)}\right) \prod_{i=1}^{n_{0}} \frac{\left(1-\zeta_{i} \theta\right)^{k_{i}}}{\left(1-\gamma_{i} \theta\right)^{p_{i}}} .
$$

\footnotetext{
${ }^{3}$ For the conciseness of the presentation, from now on the dependency of $\theta$ on $s$ will be dropped.
}

Taking the natural logarithm on both sides of the above equation leads to

$$
\begin{aligned}
\ln [L(\theta)]= & \theta \sum_{i=1}^{n_{0}} \frac{\delta_{i} \beta}{a_{i}\left(1-\gamma_{i} \theta\right)}+\sum_{i=1}^{n_{0}} k_{i} \ln \left(1-\zeta_{i} \theta\right) \\
& -\sum_{i=1}^{n_{0}} p_{i} \ln \left(1-\gamma_{i} \theta\right) .
\end{aligned}
$$

For $\theta$ satisfying $\theta<1 / \max \left\{\max _{i}\left\{\left|\gamma_{i}\right|\right\}, \max _{i}\left\{\left|\zeta_{i}\right|\right\}^{4}\right.$, where $|\cdot|$ denotes absolute value, a series expansion for $\ln [L(\theta)]$ exists and is given by

$$
\ln [L(\theta)]=\sum_{r=1}^{\infty} d_{r} \frac{\theta^{r}}{r}
$$

where

$$
d_{r}=\beta r \sum_{i=1}^{n_{0}} \frac{\delta_{i}}{a_{i}} \gamma_{i}^{r-1}-\sum_{i=1}^{n_{0}} k_{i} \zeta_{i}^{r}+\sum_{i=1}^{n_{0}} p_{i} \gamma_{i}^{r}
$$

Therefore, according to [16, pp. 93], (10) can be rewritten as

$$
L(\theta)=\sum_{r=0}^{\infty} c_{r} \theta^{r}
$$

where the coefficients of the series are computed from the simple recursive formula

$$
c_{0}=L(0) \quad \text { and } \quad c_{r}=\frac{1}{r} \sum_{l=0}^{r-1} d_{r-l} c_{l} \text { for } \quad r>0 .
$$

From (7), (10) and (14), $M_{Y}(-s)$ can be expressed in a series expansion form, i.e.

$$
M_{Y}(-s)=A \sum_{r=0}^{\infty} c_{r} \theta^{\rho+r}(s) .
$$

Therefore, using (16) and employing [17, pp. 1022, eq. (29.3.11)], $f_{Y}(y)$ for the general model described in Section II is given by the following simple Gamma series representation

$$
f_{Y}(y)=A \sum_{r=0}^{\infty} c_{r} \frac{y^{\rho+r-1} \exp (-y / \beta)}{\beta^{\rho+r} \Gamma(\rho+r)}
$$

where $A$ and $\rho$ are given by (8) and $c_{r}$ is recursively computed from (13) and (15). Applying term-by-term integration in (17), the CDF of $Y$ is expressed as follows

$$
F_{Y}(y)=A \sum_{r=0}^{\infty} c_{r} \frac{\gamma(\rho+r, y / \beta)}{\Gamma(\rho+r)}
$$

where $\gamma(\cdot, \cdot)$ is the incomplete Gamma function [18, pp. 899, eq. (8.350.1)]. In obtaining (18), uniform convergence of the series in (17) has been assumed. The validity of this assumption is established in the Appendix where the range of values of $\beta$ that ensure convergence is also obtained.

\footnotetext{
${ }^{4}$ In case $\max _{i}\left\{\left|\zeta_{i}\right|\right\}=0, \theta$ should simply satisfy $\theta<1 / \max _{i}\left\{\left|\gamma_{i}\right|\right\}$.
} 


\section{Performance Analysis}

The PDF obtained in the previous section can be conveniently used when analyzing the performance of wireless communication systems operating in different fading channels and with MRC or OSTBC type diversity. For such schemes, the instantaneous SNR at the receiver, at the presence of flat fading, is expressed as a sum of squared fading RVs. Provided that the channel fading coefficients are independent and distributed according to the models considered in Section II, general expressions can be derived for several performance analysis criteria, as will be described below.

\section{A. Capacity Analysis}

For a certain value of the SNR $Y=y$, the normalized capacity is defined as [2, pp. 115]

$$
C \triangleq R \log _{2}(1+y)
$$

where $R$ is a constant related to the adopted diversity scheme, e.g., $R=1$ for MRC, while for OSTBC, $R$ is the rate of the utilized space time block code. The capacity of a system is most frequently evaluated in terms of the information outage probability (IOP) and the ergodic capacity.

1) Information Outage Probability: The IOP, $P_{\text {out }}\left(C_{0}\right)$, is defined as the probability that a given transmission rate $C_{0}$ cannot be supported. From (19), the IOP can be expressed as

$$
P_{\text {out }}\left(C_{0}\right) \triangleq \operatorname{Pr}\left(C \leq C_{0}\right)=\operatorname{Pr}\left(y \leq 2^{\frac{C_{0}}{R}}-1\right) .
$$

Hence, the IOP can be obtained directly from the CDF of $Y$ given by (18).

2) Ergodic Capacity: The ergodic capacity $\langle C\rangle$ is defined as [2, pp. 29], i.e., from (19)

$$
\langle C\rangle \triangleq R \int_{0}^{\infty} \log _{2}(1+y) f_{Y}(y) d y
$$

Substituting (17) in the above equation yields

$$
\langle C\rangle=A R \sum_{r=0}^{\infty} c_{r} \mathcal{C}(\rho+r)
$$

where

$$
\mathcal{C}(v)=\int_{0}^{\infty} \frac{y^{v-1} \exp (-y / \beta) \log _{2}(1+y)}{\beta^{v} \Gamma(v)} d y .
$$

\section{B. Error Probability Analysis}

It is well known that if the PDF of the SNR is available, the error probability for any modulation can be calculated by evaluating integrals of the form

$$
\mathcal{P}=\int_{0}^{\infty} f_{Y}(y) P(y) d y
$$

where $P(y)$ is a modulation specific function. In the following, the SEP and BEP performance for the general model characterized by the PDF given in (17) is obtained.
1) SEP Analysis: Expressions of the SEP for various modulation schemes are first presented, by assuming that the RV $Y$ denotes the instantaneous SNR per symbol.

$M$-PAM: In this case the SEP can be conveniently written as

$$
P_{S}=A \delta \sum_{r=0}^{\infty} c_{r} \mathcal{V}(\mathrm{a}, \beta, \rho+r)
$$

where a, $\delta$ can be found from $[1$, pp. 224 , eq. $(8.3)], \mathcal{V}(\cdot, \cdot, \cdot)$ is given by [1, pp. 127 , eq. (5.17)]

$$
\mathcal{V}(a, \beta, v)=\frac{\sqrt{c / \pi} \Gamma(v+1 / 2){ }_{2} F_{1}\left(1, v+\frac{1}{2} ; v+1 ; \frac{1}{1+c}\right)}{2(1+c)^{v+1 / 2} \Gamma(v+1)}
$$

with $c=a^{2} \beta / 2$ and ${ }_{2} F_{1}(\cdot, \cdot ; \cdot ; \cdot)$ is the Gauss Hypergeometric function [17, pp. 556, eq. (15.1.1)].

$M$-QAM: Assuming square $M$-QAM modulation, the SEP can be expressed as

$$
P_{S}=2 A \delta \sum_{r=0}^{\infty} c_{r} \mathcal{V}(\mathrm{a}, \beta, \rho+r)-A \delta^{2} \sum_{r=0}^{\infty} \mathcal{H}(\mathrm{a}, \beta, \rho+r)
$$

where this time a and $\delta$ can be calculated using [1, pp. 226, eq. (8.10)], while $\mathcal{H}(\cdot, \cdot, \cdot)$ is defined as [19]

$$
\mathcal{H}(\mathrm{a}, \beta, v)=\frac{F_{1}\left(1, v, 1 ; v+\frac{3}{2} ; \frac{1+\mathrm{a}^{2} \beta / 2}{1+\mathrm{a}^{2} \beta}, 1 / 2\right)}{2 \pi(2 v+1)\left(1+\mathrm{a}^{2} \beta\right)^{v}}
$$

and $F_{1}(\cdot, \cdot ; \cdot ;, \cdot)$ denotes the Appell Hypergeometric function [20].

$M$-PSK: In this case, following a similar to the $M$-QAM approach, the SEP can be written as

$$
P_{S}=A \sum_{r=0}^{\infty} c_{r} \mathcal{V}\left(a_{P S K}, \beta, \rho+r\right)+A \sum_{r=0}^{\infty} c_{r} \mathcal{W}(\beta, \rho+r)
$$

where $a_{P S K}=\sqrt{2} \sin (\pi / M)$ and $\mathcal{W}(\cdot, \cdot)$ is defined as [19]

$$
\mathcal{W}(\beta, v) \triangleq \frac{1}{\pi} \sqrt{\eta} \mu^{v} F_{1}\left(\frac{1}{2}, v, \frac{1}{2}-v ; \frac{3}{2} ; \frac{\eta}{\mu}, \eta\right) .
$$

where $\eta=\cos ^{2}(\pi / M)$ and $\mu=1+\sin ^{2}(\pi / M) \beta$.

2) BEP Analysis: Assuming now that $Y$ corresponds to the instantaneous SNR per bit, the BEP performance for the modulation schemes previously considered follows.

$M$-PAM: In the case of Gray coded $M$-PAM, using the expression for $P(y)$ given in [21] the BEP can be written as

$$
P_{B}=\sum_{r=0}^{\infty} \sum_{j=1}^{\log _{2} M} \sum_{i=0}^{\left(1-2^{-j}\right) M-1} \frac{2 A c_{r} \delta_{M}(i, j) \mathcal{V}\left(\mathrm{a}_{i}, \beta, \rho+r\right)}{M \log _{2} M}
$$

where $\mathrm{a}_{i}, \delta_{M}(i, j)$ are given also in [21].

$M$-QAM: Recalling that the BEP of Gray coded square $M$-QAM coincides with that of Gray coded $\sqrt{M}$-PAM, it is evident that the previous results on PAM also apply here. Therefore, by substituting $M$ with $\sqrt{M}$ in (31) an expression for the BEP of square $M$-QAM can be obtained.

$M$-PSK: In the case of Gray coded $M$-PSK, a good approximation for $P(y)$ can be found in [1, pp. 234, eq. (8.32)] 
that leads to the following approximation for the BEP for $M$ PSK signals

$$
P_{B}=\sum_{r=0}^{\infty} c_{r} \sum_{i=1}^{\max (M / 4,1)} \frac{2 A \mathcal{V}\left(\mathrm{a}_{i}, \beta, \rho+r\right)}{\max \left(\log _{2} M, 2\right)} .
$$

where $\mathrm{a}_{i}=\sqrt{2 \log _{2} M} \sin \frac{(2 i-1) \pi}{M}$. To recapitulate, for the very general form of the PDF given in (17), the error probabilities for various modulation schemes have been conveniently expressed in terms of simple series expansions of the functions $\mathcal{V}(\cdot, \cdot, \cdot), \mathcal{H}(\cdot, \cdot, \cdot)$ and $\mathcal{W}(\cdot, \cdot)$ given in (26), (28) and (30), respectively. In the next section, an application is presented, where the resulting expressions for the various performance metrics will be further simplified.

\section{OSTBC over Mixed NaKagami-m/Rice Fading}

The mixed Nakagami- $m$ /Rice fading model has been considered in previous works [22],[23], to describe paths between the transmitter and the receiver, which are subject to different fading phenomena. To the best of our knowledge, however, an exact performance analysis of this model in a MIMO OSTBC environment is not available in the open technical literature. Let us consider a MIMO OSTBC system with $n_{t}$ transmit and $n_{r}$ receive antennae operating over independent fading channels. It is well known that the SNR at the receiving end can be expressed as [2, pp. 109]

$$
Y=\frac{\sum_{k=1}^{n_{r}} \sum_{l=1}^{n_{t}}\left|h_{k, l}\right|^{2} Y_{t}}{R n_{t}}
$$

where $h_{k, l}$ is the fading coefficient between the $k$-th receive and $l$-th transmit antenna and $R$ is the rate of the used OSTBC. Equivalently, $Y$ can be rewritten as

$$
Y=\sum_{i=1}^{n_{t} n_{r}} X_{i}^{2}
$$

where $X_{i}^{2}=\left|h_{k, l}\right|^{2} Y_{t} / R n_{t}$ with $k=\left\lceil i / n_{t}\right\rceil$, where $\lceil\cdot\rceil$ denotes the ceiling operator and $l=i-(k-1) n_{t}$. For this system, clearly the expressions derived in Section IV can again be conveniently used for calculating the respective performance metrics. As an example, let us consider a $2 \times 2$ MIMO system employing the Alamouti OSTBC [2, pp. 87-88]. Moreover, let us assume that $\left|h_{1,1}\right|,\left|h_{1,2}\right|$ are i.i.d. Nakagami-m RVs with parameters $m>0, \Omega_{1}$, and $\left|h_{2,1}\right|,\left|h_{2,2}\right|$ are i.i.d. Ricean distributed RVs with parameters $K$ and $\Omega_{2}$. It is then easy to show that the MGF of $Y$ becomes

$$
M_{Y}(s)=\frac{\exp \left(\delta \frac{a_{2} s}{1-a_{2} s}\right)}{\left(1-a_{1} s\right)^{2 m}\left(1-a_{2} s\right)^{2}}
$$

where

$$
a_{1}=\frac{\Omega_{1} Y_{t}}{m R n_{t}}, \quad a_{2}=\frac{\Omega_{2} Y_{t}}{(K+1) R n_{t}}, \quad \text { and } \quad \delta=2 K
$$

The Laplace transform of the PDF of $Y$ is expressed as in (7) and the PDF of $Y$ is given by (17) with

$$
A=\frac{\beta^{2(m+1)} \exp (-\delta)}{a_{1}^{2 m} a_{2}^{2}}, \quad \rho=2(m+1) .
$$

The series coefficients $c_{r}$ are computed from (15) with $c_{0}=1$ and

$$
d_{r}=\beta r \frac{\delta}{a_{2}} \gamma_{2}^{r-1}+2 m \gamma_{1}^{r}+2 \gamma_{2}^{r}
$$

for $r>0$, and $\gamma_{1}, \gamma_{2}$ given by (9). Therefore, for the performance analysis, the general capacity and error probability expressions derived in Section IV need to be employed. Notice that computation of these expressions appears complex due to the need of evaluating $\mathcal{C}(v)$ as in (23), as well as the Hypergeometric functions in (26), (28) and (30). Concerning $\mathcal{C}(v)$, the efficient computation of all respective series terms in (22) is achieved by employing a simple recursive formula. Indeed, by using simple integration techniques and applying $[18$, pp. 348 , eq. (3.383.10)] it is easy to show that

$$
\mathcal{C}(v)=\mathcal{C}(v-1)+\frac{\log _{2}(e)}{\beta^{v-1}} \exp (1 / \beta) \Gamma(1-v, 1 / \beta)
$$

where $e$ is the Neper number and $\Gamma(\cdot, \cdot)$ is the upper incomplete Gamma function [18, pp. 899, eq. (8.350.2)]. Therefore, by applying a common numerical technique to evaluate $\mathcal{C}(\xi)$ for a small $\xi$ and then using (39), it is straightforward to calculate $\mathcal{C}(v)$ for every $v{ }^{5}$ As far as the ergodic capacity is concerned, by taking $\xi$ equal to the decimal part of $\rho$, all series terms $\mathcal{C}(r+\rho)$ in (21) can be recursively computed from (39) in an efficient manner.

The Gauss and Appell hypergeometric functions are available as built-in functions in most popular mathematical software packages such as MATHEMATICA ${ }^{\mathrm{TM}}$. There also exist efficient algorithms to accurately compute these functions. For the Gauss hypergeometric function ${ }_{2} F_{1}(\cdot, \cdot ; \cdot ; \cdot)$, a computationally and numerically efficient procedure can be found in [24]. This procedure can also be used for the computation of the Appell Hypergeometric function, since it holds that [20]

$$
\begin{aligned}
F_{1}\left(a, b_{1}, b_{2}, c, z_{1}, z_{2}\right)=\sum_{r=0}^{\infty} & \frac{(a)_{r}\left(b_{1}\right)_{r}\left(b_{2}\right)_{r}(c-a)_{r}}{(c+r-1)_{r}(c)_{2 r} r !}\left(z_{1} z_{2}\right)^{r} \\
& \times{ }_{2} F_{1}\left(a+r, b_{1}+r ; c+2 r ; z_{1}\right) \\
& \times{ }_{2} F_{1}\left(a+r, b_{2}+r ; c+2 r ; z_{2}\right)
\end{aligned}
$$

where $(\cdot)_{r}$ denotes the Pochhammer symbol [17, pp. 256, eq. (6.1.22)]. Alternatively, the double infinite series expression presented in [25] can be used.

It should be noted that when $\rho$ is integer, the performance analysis is greatly simplified since the various performance metrics can be expressed in forms avoiding the use of Hypergeometric functions and numerical integration techniques.

\section{Vi. Performance Evaluation and Discussion}

In this section, Monte Carlo computer simulated results are used to verify the previously presented theoretical analysis. A communication scheme based on the scenario described in Section V is considered. It is assumed that $\left|h_{1,1}\right|$ and $\left|h_{1,2}\right|$ are i.i.d. Nakagami- $m$ RVs with parameters $\Omega_{1}=0.2$ and $m=1.3$ and $\left|h_{2,1}\right|$ and $\left|h_{2,2}\right|$ are i.i.d. Rice distributed RVs with parameters $\Omega_{2}=0.25$ and $K=4$. The convergence

\footnotetext{
${ }^{5}$ For instance, it has been verified that application of the Gauss quadrature rule given in [17, pp. 890 , eq. (25.4.45)] leads to very accurate results for small values of $\xi$.
} 


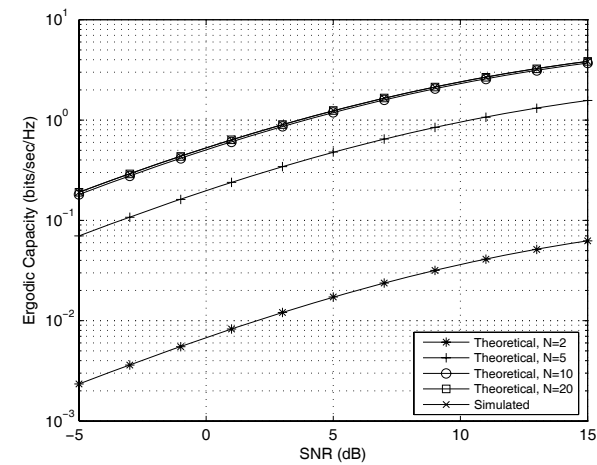

Fig. 1. Ergodic capacity as a function of the SNR.

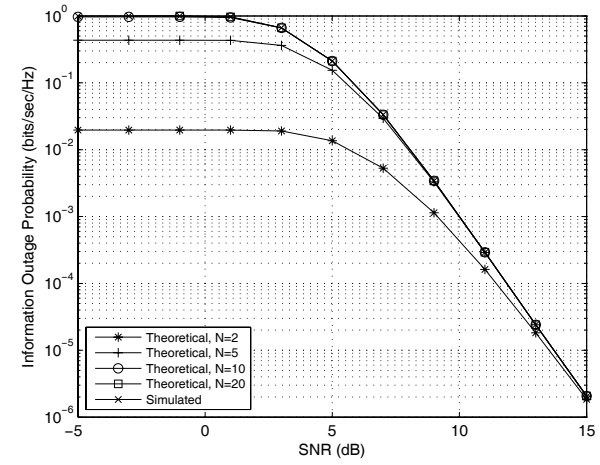

Fig. 2. Information Outage Probability as a function of the SNR.

parameter $\beta$ was taken equal to $1.7 \beta_{0}$, so that a number of 20 series terms is sufficient for accurately computing the various theoretical measures.

In Fig. 1 the ergodic capacity versus SNR is plotted for several values of the number of terms retained from (22). The theoretical curves have been obtained using (22) and (39). The accurate match of theoretical and Monte-Carlo results for $N=20$, validates our theoretical expressions and justifies the choice for $N$. In Fig. 2, theoretical and Monte-Carlo simulated results for the IOP as a function of the transmit SNR are presented for an outage transmission rate equal to $C_{0}=1$ $\mathrm{bit} / \mathrm{sec} / \mathrm{Hz}$. As observed, an excellent match between the theoretical values calculated using (20) and simulations results is achieved for $N=20$, and the accurate prediction of the performance based on the proposed theoretical analysis, can be verified. In Table II the proposed method is compared with the method presented in [13] in terms of the computational speed requirements. The time necessary for the calculation of the ergodic capacity for several values of the SNR and with an accuracy equal to $10^{-4}$ is illustrated. The methods have been implemented in MATLAB ${ }^{\mathrm{TM}}$ and were run on an Intel Dual Core Pentium processor at $2.4 \mathrm{GHz}$. Note that for the calculation of the integral in [13, eq. (4)], which is required for computing the ergodic capacity, the standard Gauss integration rule [17, pp. 887, eq. 25.4.30] has been used. It can be observed from Table II that both methods are computationally efficient, with the proposed method being less computationally demanding than the one in [13]. For each method, the slight differences in times for various SNRs are due to the different number of series terms needed to achieve the specified precision.

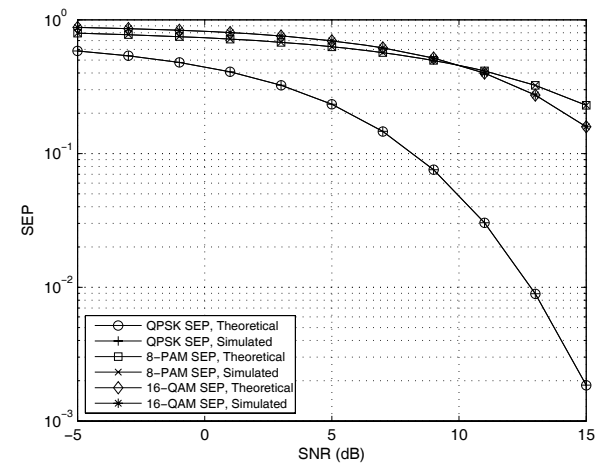

Fig. 3. Symbol Error Probability as a function of the SNR.

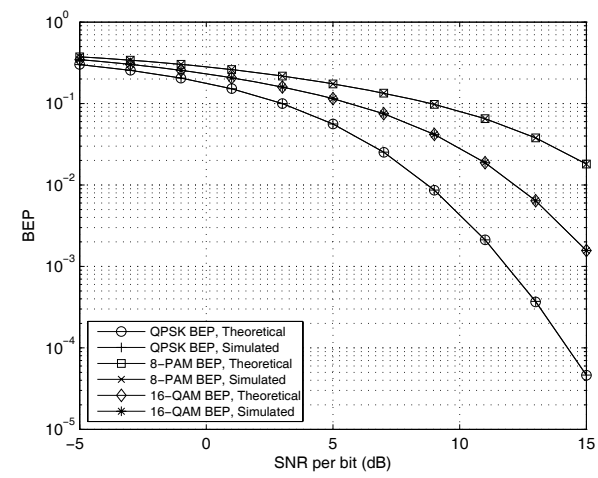

Fig. 4. Bit Error Probability as a function of the SNR.

In Fig. 3 the SEP is plotted as a function of the SNR for various modulations. Clearly the theoretical curves obtained using expressions (25) and (27) coincide with those based on Monte Carlo simulations. It is thus verified that by retaining a reasonable number of terms from the proposed series expansions, an extremely accurate calculation of the performance metrics of interest is possible. Finally, in Fig. 4 the BEP is plotted as a function of the SNR per bit. The theoretical curves have been constructed based on (31). Again the validity of the derived expressions is corroborated by the close match of theoretical and simulation results.

\section{CONCLUSION}

A novel, exact performance analysis study of MRC and OSTBC over generalized fading channels has been presented. To evaluate the performance of such diversity combining techniques, the distribution of a sum of squared RVs needs to be explored. After specifying a set of important fading distributions that share a common MGF model, a detailed statistical analysis has been presented for the sum of squared RVs belonging to this set. Based on the derived PDF and CDF expressions, an exact capacity and error probability

TABLE II

COMPARISON OF TWO METHODS IN TERMS OF THE TIME REQUIRED TO COMPUTE THE ERGODIC CAPACITY

\begin{tabular}{cccccc}
\hline \hline & & & & & \\
SNR (dB) & 1 & 3 & 5 & 7 & 9 \\
\hline Proposed (msec) & 0.73 & 0.67 & 0.67 & 0.66 & 0.68 \\
[13] (msec) & 1.9 & 1.7 & 1.8 & 2.1 & 3.3
\end{tabular}


analysis framework for MRC/OSTBC over generalized fading channels has been developed. General expressions for various performance metrics such as the IOP, the ergodic capacity and the BEP and SEP have been obtained. Among the several applications of the presented theoretical analysis, an example of an OSTBC system operating over mixed Nakagami- $m$ and Rice fading channel has been studied and simple expressions for the various performance metrics have been derived. Simulation results have been presented, which completely corroborate the proposed theoretical analysis.

\section{APPENDIX}

\section{PROOF OF CONVERGENCE FOR (17)}

The uniform convergence of (17) can be proven by bounding the series truncation error. More specifically, since $c_{r}$ 's are the coefficients of the power series expansion of $L(\theta)$, by employing Cauchy's inequality [26] in order to bound the absolute value of $c_{r}$ 's, and assuming that the first $N$ terms of (17) are retained, a bound on the absolute value of the truncation error $\varepsilon(y)$ of (17) can be obtained as

$$
\varepsilon(y) \leq A \frac{L_{0}(u) u^{\rho-1} \exp (-y / \beta)}{\beta} \sum_{r=N+1}^{\infty} \frac{(y / \beta u)^{\rho+r-1}}{\Gamma(\rho+r)}
$$

where $u$ is any positive value satisfying $u<$ $1 / \max \left\{\max _{i}\left\{\left|\gamma_{i}\right|\right\}, \max _{i}\left\{\left|\zeta_{i}\right|\right\}\right\}$ and $L_{0}(u)=\max _{|\theta|=u}|L(\theta)|$.

Closed-form expressions for the right hand side (RHS) term of (A-1) can be derived assuming that $\rho$ is not an integer. Indeed, using the definition of the Hypergeometric function $U(a, b, y)$ as found in [25, eq. 07.33.06.0002.01] for $a=b$ and property [25, eq. 07.33.03.0003.01], it is easy to show that

$$
y^{1-b} \sum_{r=0}^{\infty} \frac{y^{r}}{\Gamma(2-b+r)}=\exp (y)\left(1-\frac{\Gamma(1-b, y)}{\Gamma(1-b)}\right) .
$$

Hence, using (A-2) for $b=1-\rho-N$, the truncation error bound in (A-1) takes the form

$$
\varepsilon(y) \leq A \frac{L_{0}(u) u^{\rho-1} \exp \left(-\frac{y}{\beta}+\frac{y}{\beta u}\right)\left(1-\frac{\Gamma\left(M, \frac{y}{\beta u}\right)}{\Gamma(M)}\right)}{\beta} .
$$

where $M=\rho+N$. Moreover, the validity of (A-3) for integer values of $\rho$ can be proved using the relation between the series in the RHS of (A-1) and the exponential function as well as the property [25, eq. 06.06.03.0009.01].

From (A-3) it can be seen that, as long as $u>1$, a finite bound on the truncation error of (17) can be derived for all $y \geq 0$, which decreases with respect to $N$ and the uniform convergence is established. Hence, in order to ensure the uniform convergence, the value of $\beta$ should be chosen such that $0<\beta<2 \beta_{0}$ with $\beta_{0}$ being equal to the minimum value of the set formed by the non-zero $a_{i}$ 's and $b_{i}$ 's.

\section{REFERENCES}

[1] M. K. Simon and M.-S. Alouini, Digital Communication over Fading Channels. New York: J. Wiley and Sons, 2005.
[2] E. Larsson and P. Stoica, Space-Time Block Coding for Wireless Communications. New York: Cambridge University Press, 2003.

[3] S. Kotz, N. L. Johnson, and D. W. Boyd, "Series representations of distributions of quadratic forms in normal variables-I: central case," Annals Mathematical Statistics, vol. 38, no. 3, pp. 823-837, June 1967.

[4] — "Series representations of distributions of quadratic forms in normal variables-II: non-central case," Annals Mathematical Statistics, vol. 38, no. 3, pp. 838-848, June 1967.

[5] P. G. Moschopoulos, "The distribution of the sum of independent Gamma random variables," Ann. Inst. Statist. Math. (Part A), vol. 37, pp. 541-544, 1985.

[6] M.-S. Alouini, A. Abdi, and M. Kaveh, "Sum of Gamma variates and performance of wireless communication systems over Nakagami-fading channels," IEEE Trans. Veh. Technol., vol. 50, no. 6, pp. 1471-1480, Nov. 2001.

[7] G. A. Ropokis, A. A. Rontogiannis, and P. T. Mathiopoulos, "Quadratic forms in normal RVs: theory and applications to OSTBC over Hoyt fading channels," IEEE Trans. Wireless Commun., vol. 7, no. 12, pp. 5009-5019, Dec. 2008.

[8] K. Yip and T. Ng, "Matched filter bound for multipath Rician fading channels," IEEE Trans. Commun., vol. 46, no. 4, pp. 441-445, Apr. 1998.

[9] S. Khatalin and J. P. Fonseka, "On the channel capacity in Rician and Hoyt fading environments with MRC diversity," IEEE Trans. Veh. Technol., vol. 55, no. 1, pp. 137-141, Jan. 2006.

[10] H. Zhang and T. A. Gulliver, "Capacity and error probability analysis for orthogonal space-time block codes over fading channels," IEEE Trans. Wireless Commun., vol. 4, no. 2, pp. 808-819, Mar. 2005.

[11] R. U. Nabar, H. Bolcskei, and A. Paulraj, "Diversity and outage performance in space-time block coded Ricean MIMO channels," IEEE Trans. Wireless Commun., vol. 4, no. 5, pp. 2519-2532, Sep. 2005.

[12] Y.-C. Ko, M.-S. Alouini, and M. K. Simon, "Outage probability of diversity systems over generalized fading channels," IEEE Trans. Commun., vol. 46, no. 4, pp. 441-445, Apr. 1998.

[13] R. Palat, A. Anamalai, and J. Reed, "An efficient method for evaluating information outage probability and ergodic capacity of OSTBC system," IEEE Commun. Lett., vol. 12, no. 3, pp. 191-193, Mar. 2008.

[14] A. Abdi, W. C. Lau, M.-S. Alouini, and M. Kaveh, "A new simple model for land-mobile satellite channels: first- and second-order statistics," IEEE Trans. Wireless Commun., vol. 2, no. 3, pp. 519-528, May 2003.

[15] G. Alfano and A. D. Maio, "Sum of squared shadowed-Rice random variables and its application to communication systems performance prediction," IEEE Trans. Wireless Commun., vol. 6, no. 10, pp. 35403545, Oct. 2007.

[16] A. M. Mathai and S. B. Provost, Quadratic Forms in Random Variables: Theory and Applications. New York: Marcel Dekker, Inc., 1992.

[17] M. Abramowitz and I. E. Stegun, Handbook of Mathematical Functions with Formulas, Graphs and Mathematical Tables. New York: Dover Publications Inc., 1970.

[18] I. Gradshteyn and I. Ryzhik, Table of Integrals, Series and Products, 7th edition. Academic Press, 2007.

[19] H. Shin and J. H. Lee, "On the error probability of binary and $M$ ary signals in Nakagami- $m$ fading channels," IEEE Trans. Commun., vol. 52, no. 4, pp. 536-538, Apr. 2004.

[20] F. D. Colabvecchia, G. Gasaneo, and J. E. Miraglia, "Numerical evaluation of Appell's $F_{1}$ hypergeometric function," Comput. Physics Commun., vol. 138, pp. 29-43, 2001.

[21] K. Cho and D. Yoon, "On the general BER expression of one- and twodimensional amplitude modulations," IEEE Trans. Commun., vol. 50, no. 7, pp. 1074-1080, July 2002.

[22] F. A. Danilo-Lemoine and H. Leib, "Specular coherent and non-coherent optimal detection for unresolved multipath Ricean fading channels," IEEE Trans. Commun., vol. 50, no. 4, pp. 588-599, Apr. 2002.

[23] C.-D. Iskander and P. T. Mathiopoulos, "Exact performance analysis of dual-branch coherent equal-gain combining in Nakagami- $m$, Rician and Hoyt fading," IEEE Trans. Veh. Technol., vol. 57, no. 2, pp. 921-931, Mar. 2008.

[24] K. Mayrhofer and F. D. Fischer, "Analytical solutions and a numerical algorithm for the Gauss Hypergeometric function ${ }_{2} F_{1}(a, b ; c ; z)$," ZAMM-J. Applied Mathematics Mechanics, vol. 74, no. 7, pp. 265-273, 1994.

[25] Wolfram (2007), The Wolfram functions site. [Online]. Available: http://functions.wolfram.com

[26] G. T. Whyburn, "The Cauchy inequality in topological analysis," Proc. National Academy Sciences United States America, vol. 48, no. 8, pp. 1335-1336, Aug. 1962. 